\title{
Characterization of a mammalian prosencephalic functional plan
}

\author{
Sophie Croizier ${ }^{\dagger}$, Sandrine Chometton, Dominique Fellmann and Pierre-Yves Risold *
}

EA 3922, SFR FED 4234, UFR Sciences Médicales et Pharmaceutiques, Université de Franche-Comté, Besançon, France

\section{Edited by:}

Gonzalo Alvarez-Bolado, University

of Heidelberg, Germany

Reviewed by:

Luis Puelles, Universidad de

Murcia, Spain

Gonzalo Alvarez-Bolado, University

of Heidelberg, Germany

José L. E. Ferran, University of

Murcia, Spain

${ }^{*}$ Correspondence:

Pierre-Yves Risold, EA 3922, SFR

FED 4234, UFR Sciences Médicales

et Pharmaceutiques, Université de

Franche-Comté, 19 rue Ambroise

Paré, 25030 Besançon cedex,

France

e-mail: pierre-yves.risold@

univ-fcomte.fr

${ }^{\dagger}$ Present address:

Sophie Croizier, The Saban

Research Institute, Neuroscience

Program, Children's Hospital Los

Angeles, University of Southern

California, Los Angeles, California

90027, USA
Hypothalamic organizational concepts have greatly evolved as the primary hypothalamic pathways have been systematically investigated. In the present review, we describe how the hypothalamus arises from a molecularly heterogeneous region of the embryonic neural tube but is first differentiated as a primary neuronal cell cord (earliest mantle layer). This structure defines two axes that align onto two fundamental components: a longitudinal tractus postopticus(tpoc)/retinian component and a transverse supraoptic tract(sot)/olfactory component. We then discuss how these two axonal tracts guide the formation of all major tracts that connect the telencephalon with the hypothalamus/ventral midbrain, highlighting the existence of an early basic plan in the functional organization of the prosencephalic connectome.

Keywords: melanin concentrating hormone, cell cord, pioneer tracts, lateral hypothalamic area, medial forebrain bundle

\section{INTRODUCTION}

As a whole, the hypothalamus is involved in an extremely large range of functions, including neuroendocrine and visceral responses, thermogenesis, circadian or seasonal cycles, sleep or general arousal, the expression of specific instinctive behaviors, the control of rhythmic cortical (hippocampal) neuron firing, emotion and reward. Therefore, the hypothalamus is a complex structure composed of dozens of cell groups or nuclei that are often involved in several of these responses. Classically, the hypothalamus has been divided into four anteroposterior regions (preoptic, anterior, tuberal and posterior regions) and three longitudinal zones (periventricular, medial and lateral zones) (Swanson, 1987). This organizational scheme has been widely accepted by anatomists and physiologists during the past decades but is not satisfactory, as most of the borders are not clear and are often arbitrarily drawn. In light of anatomical findings acquired during the late 1980s and early 1990s, the organization of the hypothalamus has been revised around the concept of a behavioral control column that is composed of the medial zone nuclei (Figure 1A; Swanson, 2000, 2005). Following this new concept, each medial zone nucleus is involved in pathways that include the tectum, thalamus and telencephalon, referencing the classical circuit described by Papez in 1937 (Papez, 1995). This new view of the hypothalamic organization is interesting as it suggests that this region is fully integrated within the complex prosencephalic networks that control behavioral expression. Therefore, the hypothalamus is capable of influencing telencephalic centers, including the cerebral cortex, as well as being influenced by descending projections (Risold and Swanson, 1996, 1997; Risold et al., 1997). Conspicuous convergences have appeared between the organization of these connections and those of the classical striato-nigral and mesotelencephalic circuits, and a revision of the telencephalic organization has been proposed (Figure 1B; Risold et al., 1997; Swanson, 2000, 2003; Risold, 2004).

Although interesting, this concept primarily involves the medial zone nuclei at hypothalamic levels but ignores large sections of this structure, especially the entire hypothalamic lateral zone (lateral hypothalamic area, LHA) (Figure 1A). The LHA is a poorly differentiated region that has always been viewed as a rostral extension of the brainstem reticular formation or a bed nucleus of the medial forebrain bundle $(\mathrm{mfb})$. The $\mathrm{mfb}$ is the major fiber tract of the basal prosencephalon that passes through the LHA and bidirectionally connects more than fifty cell groups in the brainstem and telencephalon (Nieuwenhuys et al., 1982; Swanson, 1987). The mfb is a specific attribute of the LHA 


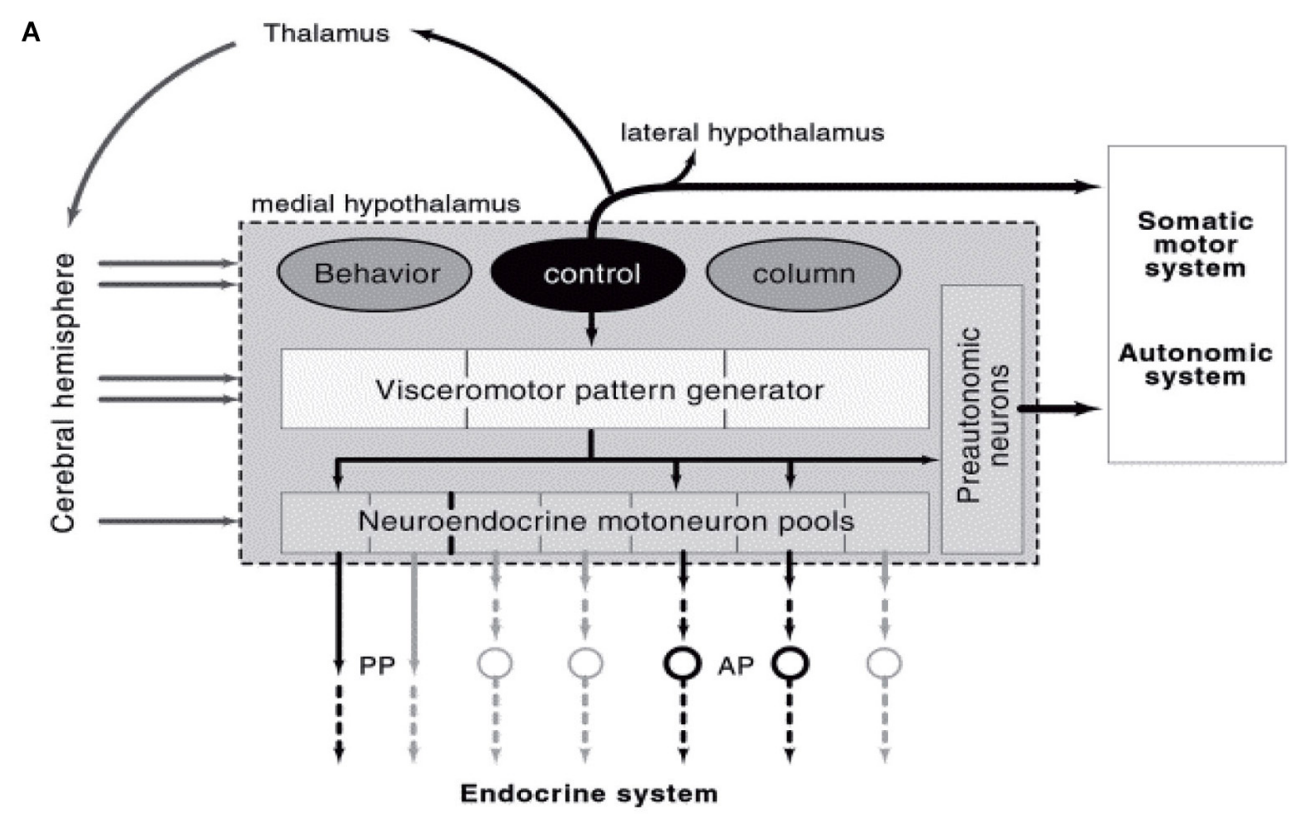

B


BRAINSTEM

FIGURE 1 | (A) Diagram illustrating the recent concept of the morphofunctional organization of the hypothalamus. Hypothalamic periventricular structures form a visceromotor pattern generator (VMPG) network that controls neuroendocrine and visceral responses. This VMPG is influenced by medial zone nuclei. These nuclei form a behavioral control column and are involved in the expression of goal oriented behaviors. They control somatic motor responses through projections in the tectum (periaqueductal gray), but they are also involved in closed loop circuits with the telencephalon, in particular through projections to the thalamus. Reproduced with permission from Thompson and Swanson, 2003.

(B) Schematic representation of the organization of the telencephalon (adapted from Risold, 2004). The cerebral cortex as a whole, including the cortico-amygdaloid nuclei, topographically projects onto the striatum (dorsal, ventral, medial, and posterior divisions), which is connected with the pallidum (similarly parceled into dorsal, ventral, medial and posterior divisions). While projections from the cortex are glutamatergic, the striatum and pallidum are massively GABAergic and are bidirectionally connected with the brainstem. Abbreviations: AP: anterior pituitary; BST: bed nuclei of the stria terminalis; CEA: central nucleus amygdala; Cortico Amy: cortical nuclei amygdala; GABA: gamma aminobutyric acid; Glu: glutamate; LSC: lateral septal complex; MEA: medial nucleus amygdala; MSC: medial septal complex; Pal d-v-m-c: dorsal, ventral, medial and caudal divisions of the pallidum; PP: posterior pituitary; SI: substantia inominata; Str d-v-m-c: dorsal, ventral, medial and caudal divisions of the striatum. throughout its entire alar-basal extent. However, the LHA is not homogeneous. The basal portion of the LHA contains abundant cell populations that are characterized by the expression of specific peptides, such as melanin-concentrating hormone $(\mathrm{MCH})$ and hypocretins/orexins, and widespread projections from the cerebral cortex to the spinal cord.

Tremendous progress in expanding the general knowledge of the forebrain embryonic development has been made over the last 
twenty years. Recent studies, alongside anatomical data, have led to a better understanding of the organization of the vertebrate forebrain, which has allowed for a better comprehension of its evolution (Puelles, 2001; Aboitiz, 2011). To understand the organization of the basal portion of the LHA, our group analyzed the comparative anatomy and development of hypothalamic neurons that produce $\mathrm{MCH}$ (Croizier et al., 2013). In the present analysis, we revised some of these observations from past and recent developmental studies regarding the forebrain to better understand the relative role of the LHA within the context of a putative general prosencephalic framework. We observed that the whole ventral prosencephalon is organized around a precocious structure, previously named the cell cord and from which the LHA differentiates, in a timely manner. This primary structure defines two axes that align onto two fundamental components: a tractus postopticus (tpoc)/retinian component and a supraoptic tract (sot)/olfactory component. These two axes determine the path of the $\mathrm{mfb}$ and provide what can be described as a basic structural framework for a prosencephalic "functional plan".

\section{DEVELOPMENTAL GENE PATTERNS AND HYPOTHALAMIC SUBDIVISIONS}

Very complex molecular interactions occur at the origin of the hypothalamic regions, which are very heterogeneous, even at the earliest stages. These patterns have been extensively analyzed in many more detailed works to which the reader may refer (Shimamura et al., 1995; Nieuwenhuys et al., 1998; Puelles et al., 2012). Longitudinal and transverse axes in the embryos have been revised on the basis of these patterns of gene expression (Puelles et al., 2012). Although the terminology proposed by Puelles and Rubenstein is widely used in the developmental field (see for instance "prethalamus"), in the field of adult neuroanatomy the traditional axes and nomenclature are maintained. Since we strive to be clear for all interested readers, "adult" or "developmental", we often hesitate to use one or the other name for a structure or spatial relation. For this practical reason, and in particular to respect the main information flows in the adult brain, we have used sometimes similar rostrocaudal and dorsoventral axes in the embryonic brain as are used in the adult brain (for example see in Figure 3). We also use the terminology prethalamus-ventral thalamus, in this way adding "prethalamus", as is often done in developmental studies, for the presumptive regions of the zona incerta and the ventral lateral geniculate nuclei. The preoptic region (POA) (actually a part of the telencephalon, see below), the anterior region (or alar hypothalamus) that is supraoptic and regions that are posterior (basal) or postoptic represent no problem (Figure 2).

From the general literature in this field one important observation retained our attention: very early in development, these gene expression patterns bear resemblances to and are contiguous with those in structures adjacent to the hypothalamus, which suggests that some hypothalamic boundaries are not sharply delineated (Figure 2A). Consider the following examples.
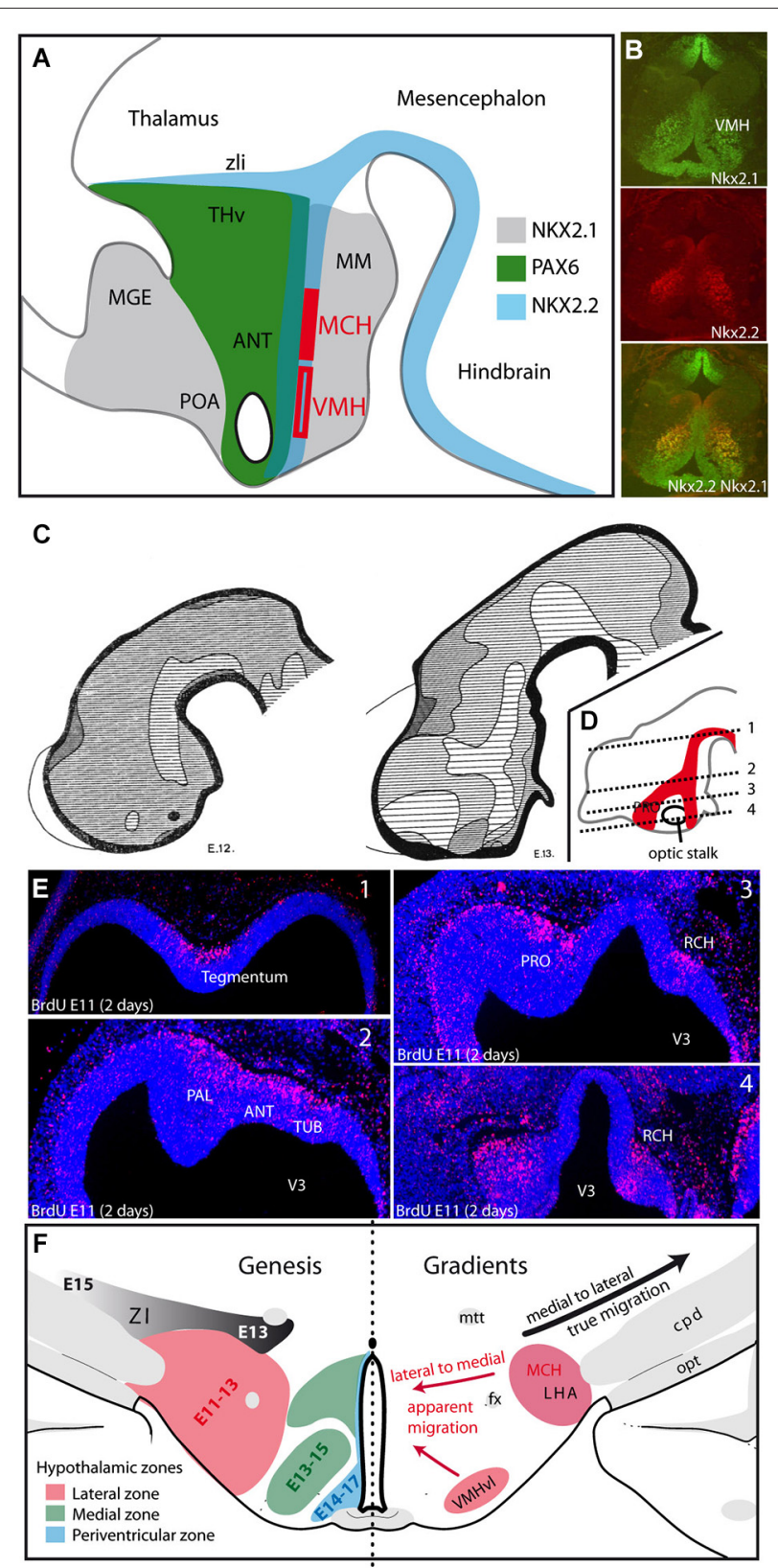

FIGURE 2 | (A) The distribution patterns of transcription factors agree well with the alar-basal divisions of the hypothalamus into preoptic, anterior and posterior/postoptic regions. However, these patterns extend outside the borders of the hypothalamus, involving the ventral telencephalon, prethalamus-ventral thalamus and ventral midbrain. $\mathrm{MCH}$ neurons and

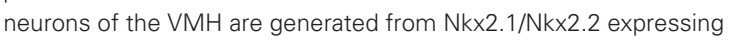
neuroepithelial zones in the postoptic region. (B) Based on immunohistochemical analysis of a horizontal section of an E15 rat embryonic hypothalamus, the $\mathrm{VMH}$ clearly express both $\mathrm{Nkx2.1}$ and Nkx2.2. (C) Figures from Keyser (1972) illustrating the differentiation of the early neurogenic zone in E12 and E13 Chinese hamster embryos: the first appearance of a longitudinal zone in the ventral mesencephalon and dorsal hypothalamus correspond to the cell cord (A). At E13, neurogenesis involved larger regions in the preoptic/ventral telencephalon; in other figure from Keyser that is not shown here, this author observed these regions forming one single continuum (as in $\mathbf{D}$ ). This continuum takes the shape of

(Continued) 


\section{FIGURE 2 | Continued}

an inverted Y. (D,E) Figure adapted from Croizier et al., 2011 illustrating the distribution of neurons generated at E11 on an E13 rat embryo. BrdU was injected into the pregnant dam at E11, and embryos were taken 2 days later at E13. BrdU was detected by immunohistochemistry on horizontal sections. The distribution pattern of these nuclei is schematized on a sagittal section in (D). BrdU-labeled nuclei follow an inverted $Y$ pattern. In (E) pictures are arranged from dorsal (1) to ventral (4). (F) Gradients of neurogenesis in the ventral diencephalon. Left side: Schematic representation of the neurogenic gradients in the hypothalamus and prethalamus-ventral thalamus, as described by Altman and Bayer (1986). The LHA is generated between E11and E13, the medial hypothalamus from $\mathrm{E} 13$ to $\mathrm{E} 15$ and the periventricular zone from E14 to E17. Note the medial to lateral gradient in the prethalamus-ventral thalamus (generated from E13 to E15). Right side: Drawing summarizing the gradients in the ventral diencephalon: lateral to medial gradients (red arrows) in the hypothalamus suggest the apparent or passive migrations of neurons in lateral territories (LHA or VMHvl), but the prethalamus-ventral thalamus requires the effective migration of cells away from the ventricular surface (black arrow). Abbreviations: ANT: anterior hypothalamic area; cpd: cerebral peduncle; fx: fornix; LHA: lateral hypothalamic area; $\mathrm{MCH}$ : melano-concentrating hormone expressing neurons; MGE: medial ganglionic eminence; MM: mammillary body; mtt: mammillothalamic tract; opt: optic tract; PAL: pallidum; POA: preoptic area; PRO: presumptive preoptic area; $\mathrm{RCH}$ : retrochiasmatic region; THv: prethalamus or ventral thalamus; TUB: presumptive tuberal hypothalamic region; $\mathrm{VMH}$ : ventromedial hypothalamic nucleus; VMHvl: ventrolateral part of the VMH; ZI: zona incerta; zli: zona limitans intrathalamica; V3: third ventricle.

\section{THE HYPOTHALAMIC/TELENCEPHALIC BORDER}

The POA shares many characteristics and developmental expression patterns with the pallidum, and has been considered a telencephalic structure (Moreno and González, 2011; Puelles et al., 2012). The preoptic anlage expresses the telencephalic marker Foxg1, distinguishing it from hypothalamic structures. Shh and Nkx2.1 are co-distributed in the POA, but their expression patterns extend into the pallidal anlage. Although the POA expresses Shh, it does not express Nkx2.2, whereas the hypothalamic regions do express Nkx2.2. Defining the telencephalo-hypothalamic limit has always been problematic, despite functional and connectional studies in adult animals that have been interpreted in favor of including the POA as part of the hypothalamus (Swanson, 1987; Risold et al., 1997). Earlier authors often viewed this region to be a medial and unevaginated section of the telencephalon or telencephalon impar (His, 1893; Herrick, 1910; Kuhlenbeck, 1929). These early claims have been strengthened by recent studies of gene expression patterns (Moreno and González, 2011). This definition is further reinforced by recent findings that, similar to the lateral and medial ganglionic eminences (LGE and MGE, respectively), the POA produces GABAergic (gamma aminobutyric acid) interneurons that tangentially migrate into the cerebral cortex (Brown et al., 2011; Gelman et al., 2011; Vitalis and Rossier, 2011). In addition, migrating cells from the olfactory epithelium colonize the medial septal and preoptic areas. Many of these cells express GnRH (gonadotropin-releasing hormone) and are neuroendocrine neurons, including in the septal region. Neuroendocrine functions are a hallmark of the hypothalamus, but a putative neuroendocrine zone extends beyond the actual rostral border of the hypothalamus. Therefore, a putative hypothalamo/telencephalic limit at the septal/preoptic border is not delineated by the distribution pattern of neuroendocrine GnRH neurons, the origin of GABAergic interneurons migrating into the pallium, or developmental gene expression patterns.

\section{ANTERIOR HYPOTHALAMUS/PRETHALAMUS-VENTRAL THALAMUS}

At early embryonic stages, Pax6 is expressed in a continuum that includes the presumptive prethalamus-ventral thalamus and extends into the optic vesicle (Stoykova et al., 1996; Puelles et al., 2013). In the hypothalamus, this pattern involves a strip of tissue between the optic stalk and the prethalamus-ventral thalamus (Figure 2A). The Pax6 expression pattern (encompassing the alar hypothalamus and prethalamic eminence) clearly suggests an elongation of the primary embryonic brain (see the interesting paper of (Suzuki et al., 2014) about the rise of the eyes in chordates). As previously noted, Pax family members are involved in the formation of the retina and are also involved in the guidance of retinal projections and the differentiation of retinorecipient structures into the suprachiasmatic nucleus (SCN). In vertebrates, such as lampreys or batrachians, the $\mathrm{SCN}$ is adjacent to the prethalamus. In the anuran, Dominguez confirmed this close and continuous positioning of the anterior hypothalamus and prethalamus (Dominguez et al., 2013). In some species, projections from the prethalamus and from the tectum reach the retina through the optic tract. In mammals, the ventral geniculate body has retained a strong retinal afferent, and the intergeniculate leaflet, a small structure of prethalamicventral thalamic origin, has strong bidirectional connections with the SCN, reminiscent of the adjacent positions of the SCN and the prethalamus in non-amniote vertebrates (see the recent work of Suzuki et al., 2014 as well). Therefore, early Pax6 expression patterns prefigure optic related pathways that in the alar hypothalamus and in the prethalamus-ventral thalamus.

\section{THE HYPOTHALAMUS AS A ROSTRAL STRUCTURE}

Also of particular interest for the development of the hypothalamus are the longitudinal expression patterns of Shh and Nkx2.2, which label a band of hypothalamic neuroepithelial tissue that rostrally extends from a similar band in the ventral mesencephalon (Shimamura et al., 1995; Alvarez-Bolado et al., 2012). When the anterior neuropore closure occurs, the initial expression patterns of Shh and Nkx2.2 involve the differentiating zona limitans intrathalamica (zli), at the junction between the prethalamus and thalamus. At roughly the same stage, corresponding to the beginning of neurogenesis, Shh expression (but not Nkx2.2) appears in a telencephalic region. The domain of Nkx2.2 overlaps partially with both Pax6 and Nkx2.1 expression domains (Croizier et al., 2011). The Pax6/Nkx2.2-rich region gives rise to anterior hypothalamic structures whose composition in the adult are not yet completely clear. However, the Nkx2.1/Nkx2.2 regions give rise to vast portions of the basal hypothalamus. We have clearly shown (Croizier et al., 2011) that neurons that produce $\mathrm{MCH}$ are generated and differentiate under the control of Shh (Szabó et al., 2009; Alvarez-Bolado et al., 2012) in this sector of the embryonic wall. In the model of Puelles, this is the RTu-I portion of the basal hypothalamus (Puelles et al., 
2012); in this way, the MCH cells would represent a precocious peduncular superficial derivative of the dorsal retrotuberal basal domain.

The ventromedial hypothalamic nucleus is produced by a more rostral portion of the $\mathrm{Nkx} 2.1 / \mathrm{Nkx} 2.2$ region (Figure $2 \mathrm{~B}$, and see (Altman and Bayer, 1986) for the origin of this nucleus). Shimogori identified this region as the "intrahypothalamic diagonal", on the basis of multiple gene expression patterns ("diagonal", that is, neither columnar nor prosomeric, but somewhat in the middle of both, rather confusingly) (Shimogori et al., 2010). The Nkx2.2 and Shh expression patterns extend into the brainstem and are known to be involved in the genesis of other very early defined neurons, including serotonergic neurons, which have diffuse projection patterns similar to $\mathrm{MCH}$ neurons (Ye et al., 1998). Somewhat later, Shh is also involved in the differentiation of dopaminergic ventral midbrain neurons (Riddle and Pollock, 2003; Perez-Balaguer et al., 2009). Therefore, the co-expression of two primary markers of the basal neural tube extends into the postoptic (i.e., basal) hypothalamus, and we observe the early production of specific neuron populations with diffuse projection patterns as $\mathrm{MCH}$ and serotonergic neurons in corresponding hypothalamic regions and hindbrain.

The mammillary nuclei and regions of the very ventromedial hypothalamus (VMH, arcuate nucleus) are generated by an Nkx2.1 expressing neuroepithelial zone (Puelles and Rubenstein, 2003). This appears to be the only pattern that does not show any sign of extension outside of the hypothalamic borders (although, see Puelles et al., 2013).

From all these observations we can conclude that the hypothalamus has diverse origins. Patterns of gene expressions are very complex, and precise combinations of gene expression are associated with specific cell groups or nuclei. However, at the very early stages, the patterns of Shh, Pax6, Nkx2.2 and Nkx2.1 expression indicate that the POA is a part of the telencephalon (Puelles et al., 2012) and the anterior (alar hypothalamus) and postoptic regions (basal hypothalamus) share some gene expression patterns with the prethalamus and the ventral (basal) brainstem.

\section{EARLY NEUROGENESIS IN THE \\ HYPOTHALAMUS-EVIDENCE OF A PRIMARY STRUCTURE}

In the embryonic neural tube, neurogenesis (neuron production and therefore the formation of a postmitotic "mantle layer") begins in the ventral hindbrain, behind the cephalic flexure. This neurogenic zone extends both caudally and rostrally. In more rostral regions, Keyser very precisely depicted the patterns of morphologic modifications that occur in the hypothalamic periventricular and mantle layers of the Chinese hamster (Keyser, 1972), describing the development of a "matrix" that can be translated into a very dynamic view of the pattern of neurogenesis in the diencephalon (Figure 2C, right and left diagrams). These observations by Keyser can easily be correlated with neurogenesis studies using tritiated nucleotides or BrdU. Keyser showed that the early pattern of neuron production is not uniform throughout the hypothalamus. Neurogenesis begins in a column of cells that was named the "cell cord" by Gilbert in 1935 in the human embryo (cited in Keyser, 1972), and it was more recently observed again in the mouse embryo by Croizier (Croizier et al., 2011; Figures 2D,E). The position of the cell cord on the model of Puelles is probably basal, immediately under the alar-basal boundary (Puelles et al., 2012). This column of early neurogenesis gives rise to the first generated hypothalamic neurons that ultimately form the postchiasmatic lateral hypothalamus, and extends into the ventral midbrain. $\mathrm{MCH}$ expressing neurons are among the first generated cells in this region, and we showed their early differentiation within this cell cord (Croizier et al., 2011). From this original sector, neurogenesis involves more rostral territories (presumptive entopeduncular nucleus (Altman and Bayer, 1986)). Therefore, the early mantle layer forms the shape of an inverted $\mathrm{Y}$ in the hypothalamic primordium (Figures 2C,D). The vertical (postoptic) limb and stem of the $\mathrm{Y}$ are longitudinal and correspond with the Shh/Nkx2.2/Pax6 expression patterns. The supraoptic arm, however, does not respect these longitudinal patterns and is transversally oriented (longitudinal and transverse here in the sense of the model by Puelles et al., 2012). A chronological correlation could be made between the development of this supraoptic arm and the differentiation of the zli (another transverse feature), the differentiation of the telencephalic vesicle or the telencephalic expression of Shh. However, causal links have not yet been demonstrated.

Keyser also observed that the development of the mantle layer extends from these hypothalamic initial regions, in both the ventral (hypothalamic) and dorsal (ventral thalamic/prethalamic) directions (Keyser, 1972). Altman and Bayer described a lateral to medial gradient of neurogenesis in the hypothalamus (lateral to periventricular) that is clearly in agreement with the observations of Keyser (Figure 2F; Altman and Bayer, 1986). A similar gradient was also clearly demonstrated for $\mathrm{MCH}$ expressing neurons (Brischoux et al., 2001; Croizier et al., 2010). On the contrary, both Altman and Bayer (1986) in the rat, and Keyser (1972) in the Chinese hamster, described a medial to lateral gradient of neurogenesis in the prethalamus that generates the zona incerta (adult ventral thalamus). This gradient is opposite to the hypothalamic gradient, although both structures are generated during the same period (between E11 and E16 in the rat). Therefore, these two opposing gradients involving two adjacent structures, lateral to medial for the hypothalamus and medial to lateral for the zona incerta, clearly designate a unique sector of origin, and the region giving rise to the cell cord is a good candidate. The opposite (lateral-medial and medial-lateral) gradients of genesis in the hypothalamus and prethalamus-ventral thalamus suggest distinct strategies of cell migration; the lateral to medial hypothalamic gradient suggests a dominant passive migration, as was shown for $\mathrm{MCH}$ expressing neurons in the dorsal hypothalamus and is also evident for the VMH. However, the medial to lateral gradient of the zona incerta indicates that lateral neurons must actively migrate far from the ventricular surface (Figure 2F; Keyser, 1972; Altman and Bayer, 1986).

Therefore, the initial neurogenesis in the hypothalamus produces a primary inverted Y-shaped structure named here the cell cord (although this structure is slightly different but encompasses the original cell cord). The prethalamus-ventral 
thalamus and medial hypothalamus are then produced through inverted gradients that are dorsal and ventromedial to this initial cell cord, respectively.

\section{THE STRUCTURAL WIRING OF THE HYPOTHALAMUS AND THE CHRONOTOPIC DIFFERENTIATION OF THE WHOLE PROSENCEPHALON}

Gene expression patterns indicate that the development of the hypothalamus is a multifactorial process, but gradients of neurogenesis show that time is a key parameter. The study by Altman and Bayer (1986) emphasized this point when these authors described three waves of genesis to form the three longitudinal zones of the hypothalamus, even if "three waves" might not to be literally considered (Alvarez-Bolado et al., 2012). Time is also a critical parameter according the description of the "matrix" (mantle layer) and cell cord by Keyser (1972). Relying on the development of $\mathrm{MCH}$ expressing neurons, we have more recently illustrated the importance of chronology in the organization of this conspicuous neuron population in the posterior hypothalamus in both rat and mouse (Croizier et al., 2010, 2011). Therefore, an analysis of the hypothalamic wiring is inseparable from the timely and sequential events that lead to the morphofunctional organization of the whole prosencephalon.

Tract formation in the hypothalamus immediately follows neurogenesis and accompanies the differentiation of hypothalamic regions. The main axonal bundles of the prosencephalon, transverse and longitudinal, have been illustrated on the prosomeric model by Puelles et al. (see their Figure 8.34) (Puelles et al., 2012). Pioneer tracts have been well described in a series of papers (Herrick, 1910; Easter et al., 1993; Mastick and Easter, 1996). Pioneer tract organization is very well conserved in the young embryo of all vertebrates, from fishes to mammals. The tpoc is the first prosencephalic tract. It is composed of commissural axons (postoptic commissure) and axons running toward the ventral midbrain, parallel to the Nkx2.2 expression domain. This tract joins the medial longitudinal fasciculus (mlf) in the midbrain. The sot and the stria medullaris are formed in the preoptic/entopeduncular primordium. The sot joins the tpoc by passing over the optic stalk (Anderson and Key, 1996), while the stria medullaris runs toward the dorsal diencephalon. The long projections of the hypothalamus subsequently organize along these pioneer tracts, and several stages can be recognized. Each stage is correlated with a different degree of organization in the embryonic brain and is reflected in the structure of the adult hypothalamus.

- The first stage is concerned with the initial formation of the pioneer tracts. Guidance cues, such as Slit/ROBO family members, and transcription factors, such as Pax6, play important roles in constraining the paths of pioneer tracts (Mastick et al., 1997; Nural and Mastick, 2004; Ricaño-Cornejo et al., 2011). Both tpoc and sot clearly recall the early neurogenic pattern and travel along the inverted Y-shaped cell cord.

Initially, these tracts are composed of descending axons. The first $\mathrm{MCH}$ expressing neurons and the first neurons in the ventrolateral $\mathrm{VMH}$ are generated during this preliminary stage (Figure 3A). MCH and SF-1 are expressed in neurons within the dorsal and ventral cell cords, respectively, and their axons have been traced in the tpoc running toward the mesencephalon (Croizier et al., 2011; Cheung et al., 2013). In the adult hypothalamus, this first stage is represented by spinally projecting $\mathrm{MCH}$ neurons (Brischoux et al., 2001; Croizier et al., 2010, 2011) that are located very laterally in the rat LHA (Figure 3E). Neurons in the lateral region of the adult $\mathrm{VMH}$, where the first SF1-labeled cells settle, send abundant projections through the supraoptic commissures (Canteras et al., 1994), and this pattern is also clearly reminiscent of descending SF-1 projections in the tpoc.

- The second stage is characterized by the growth of ascending projections along the tpoc and sot. This growth is particularly well illustrated by the differentiation of ascending projecting $\mathrm{MCH}$ expressing neurons (Figure 3A). During this stage, large bundles of ascending axons containing neurotransmitters, such as serotonin and dopamine, develop from the brainstem. The projections from hindbrain serotonergic or ventral midbrain dopaminergic neurons are initially longitudinal as they follow the tpoc, changing course in the basal hypothalamus and becoming transversally oriented in order to migrate towards the telencephalon (Figures 3B-D). $\mathrm{MCH}$ expressing neurons settle in the hypothalamic region, where these axons change direction. Moreover, in the rat, the phenotype of $\mathrm{MCH}$ expressing neurons change drastically as the mesotelencephalic dopaminergic pathway develops. As mentioned above, the first $\mathrm{MCH}$ expressing neurons send descending axons to the spinal cord. However, $\mathrm{MCH}$ expressing neurons produced during the second stage, as the dopaminergic mesotelencephalic axons progress in the $\mathrm{mfb}$, project axons toward the telencephalon but not the spinal cord in the adult rat (Brischoux et al., 2002; Croizier et al., 2010, 2011; Figure 3E).

The mechanisms responsible for the change in the axial organization of the $\mathrm{MCH}$ population appear to be related to the differentiation of the telencephalic vesicles (Croizier et al., 2011). The growth and differentiation of telencephalic vesicles involves complex interactions between morphogenic molecular actors, such as Fgf8 and Wnts (Rubenstein et al., 1998; Aboitiz, 2011). These proteins are produced by organizing centers and may diffuse and act far from their production sites. Croizier also detected a sharp increase in the production of the chemoattractant Netrin 1 in the telencephalon following the onset of neurogenesis (Croizier et al., 2011). Therefore, the telencephalon exerts a strong influence on the developing rostral brainstem as it differentiates. This influence likely increases as the rostral brainstem becomes involved in very active neurogenesis (Figure 4A). Although not fully understood, the telencephalic organizing centers, such as the ventral and cortical hems, and the diencephalic organizing centers, such as the zli, interact through the production of morphogenic protein gradients (Marín et al., 2002; Pottin et al., 2011; Rash and Grove, 2011). These processes are important for the coordinated growth of the cortex and thalamus and for the establishment 

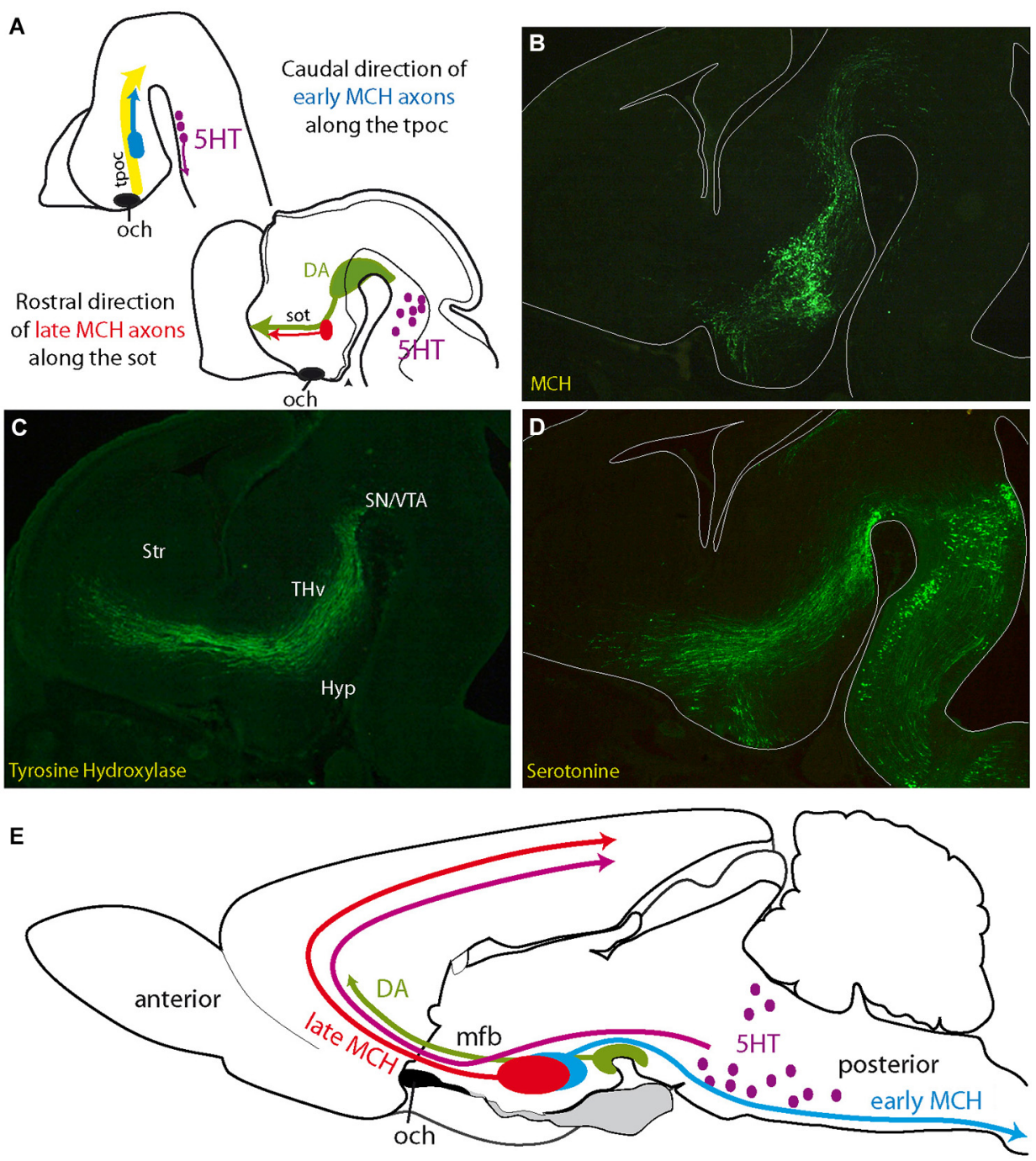

FIGURE 3 | (A) Initial projections of MCH expressing neurons follow pioneer tracts, but their direction changes as the embryo matures. Axons of first generated $\mathrm{MCH}$ expressing neurons follow the tpoc toward the midbrain. Axons from late generated $\mathrm{MCH}$ expressing neurons follow the sot toward the telencephalon, along DA and 5HT axons (see Croizier et al., 2011).

(B-D) Distribution of serotonin, $\mathrm{MCH}$ (MCH-GFP, revealed with an anti-GFP antibody; see Croizier et al., 2011) and tyrosine hydroxylase (dopamine) in three adjacent sections cut in the parasagittal plane and passing through the mfb of an E14 mouse embryonic brain. Serotonergic and dopaminergic axons from respectively the hindbrain and midbrain travel along the tpoc and arch rostrally at the level of the posterior hypothalamus, where $\mathrm{MCH}$ expressing cells are found. Note the pattern of serotonergic axons that closely follows the inverted $Y$ pattern of the sot/tpoc. (E) Schematic illustration of the ascending serotonergic and dopaminergic pathways to the telencephalon through the $\mathrm{mfb}$ and the distribution patterns of early and late $\mathrm{MCH}$ projections in the adult rat central nervous system. Abbreviations: DA dopaminergic neurons; Hyp: hypothalamus; $\mathrm{MCH}$ : melano-concentrating hormone containing neurons; mfb: medial forebrain bundle; och: optic chiasm (or presumptive position of the optic tract in $\mathbf{A}$ ); SN: substantia nigra; sot: supraoptic tract; Str: presumptive striatal region; THv: ventral thalamic region; tpoc: tractus postopticus; VTA: ventral tegmental area (presumptive); $5 \mathrm{HT}$ : serotonergic neurons. of corticothalamic and thalamocortical connections. However, the second stage also corresponds to an outburst of neurogenesis throughout the hypothalamus and the ventral mesencephalon. The dopaminergic neurons of the substantia nigra and ventral tegmental area are representative of this second stage. Their soma migrate through pre-existing premotor or motor midbrain structures, such as the Edinger-Westfall or oculomotor nuclei, to settle in the ventral midbrain. Their ascending projections through the lateral $\mathrm{mfb}$ to the striatum define them anatomically. The outburst of neurogenesis in the hypothalamus includes cortically projecting $\mathrm{MCH}$ expressing neurons and most of the hypothalamic medial zone that is generated between E13 and E15 in the rat (Altman and Bayer, 1986). During the same stage, Cheung reported ascending SF-1 expressing axons from the $\mathrm{VMH}$ in the $\mathrm{mfb}$ (Cheung et al., 2013).

Therefore, the second stage in the development of the hypothalamus is characterized by the differentiation of most of the hypothalamic lateral and medial cell groups and is concomitant with the differentiation of the dopaminergic ventral midbrain. 
- The third stage corresponds with the differentiation of the thin periventricular hypothalamic zone and neuroendocrine pathways, as neurogenesis reaches exhaustion in the hypothalamic anlage. Most of the late produced periventricular structures are regions of the visceromotor pattern generator (VMPG) that was described by Thompson and Swanson (Thompson and Swanson, 2003). These structures are often perichiasmatic or involve dorsal hypothalamic cell groups (dorsomedial nucleus, dorsomedial capsule of the $\mathrm{VMH}$ ). The last generated $\mathrm{MCH}$ expressing neurons in the rat are periventricular and project to the arcuate nucleus (Croizier et al., 2010), and some others can be characterized as neuroendocrine in nature (Cvetkovic et al., 2003). These last produced MCH expressing neurons can be viewed as part of the VMPG. Therefore, most of the VMPG and LHA could have a common origin in the periventricular dorsal hypothalamus but be generated at different periods.

- Finally, perinatal processes that are not further evocated here and that are associated with the functional organization of the hypothalamus might constitute a fourth stage. For example, the morphological modifications induced by hormones, such as sexual steroids or leptin, are now well described during the perinatal period (Bouret et al., 2004; Simerly, 2005; Ciofi et al., 2007).

\section{HYPOTHALAMIC TRACT TOPOGRAPHIC ORGANIZATION}

The anatomical dispositions of all the major tracts crossing or bounding the hypothalamus of the adult animal are summarized in Figure 4B and supplementary information. From this, it appears that all of the major tracts that originate in the dorsal or ventral telencephalon or in the retina converge in the anterior hypothalamic region (alar hypothalamus). However, upon reaching the caudal hypothalamus, they all follow adjacent pathways. This topographical organization closely follows the initial scaffolding provided by the tpoc in the caudal hypothalamus, as well as that provided by the sot for descending tracts from the telencephalon. The more ventral of these tracts (fornix, stria terminalis, ventral lateral hypothalamic tract, neuroendocrine tracts) end in the neurohypophysis and caudal hypothalamus, while the others ( $\mathrm{mfb}$, cerebral peduncle) take divergent routes at the mesencephalic limit. The relative path of these tracts can be illustrated on a schematic sagittal view of the embryonic brain (Figure 4C). We therefore observed that the descending tracts from the telencephalon traveled along a transverse path related to the sot, but as they joined the optic tract at the level of the posterior hypothalamus, their course became longitudinal, coincident with that of the tpoc and optic tract. Only the stria medullaris escaped from this general scheme.

\section{STRUCTURAL ORGANIZATION OF THE HYPOTHALAMUS AND THE PROSENCEPHALIC FUNCTIONAL PLAN}

The development of the hypothalamus and the adjacent "prethalamus-ventral thalamus" involves several stages. The first stage includes the differentiation of the cell cord. The inverted Y-shaped arrangement is the first differentiated structure of the prosencephalon and guides the first pioneer tracts, including the tpoc and sot. Following the differentiation of the cell cord, neurogenesis becomes generalized in the prosencephalon, preceding the formation of all of the major fiber tracts that connect the telencephalon with the hypothalamus and mesencephalon. Therefore, as new neurons settle medially or dorsally to the cell cord, fiber tracts topographically organize dorsally or medially to the early mfb. The tpoc also guides the optic tract and the supraoptic commissures. Therefore, while the supraoptic arm of the initial inverted Y-shaped pattern appears to guide the olfactory projections in the hypothalamus, the postoptic guides tracts parallel to retinal projections (Figure 5A).

These first and second stages in the forebrain differentiation leave traces that are found in the adult anatomical organization of the hypothalamus. In the adult brain, neurons that were initially derived from the cell cord form a large part of the reticularly organized hypothalamus, including the LHA. This structure can be considered, at a functional level, a rostral extension of the primary medial mantle layer that originates in the hindbrain and from which serotonergic neurons are produced. The concept of a deep structure in the brain with a reticular like appearance that is involved in general arousal in all vertebrates and that forms a reticular core is quite ancient. For example, this reticular core was termed the isodentritic core by Ramon-Moliner and Nauta and was also compared to the deep ancient brain described by McLean (Ramón-Moliner and Nauta, 1966; Nieuwenhuys et al., 1998; Swanson, 2003). Pfaff recently argued that primitive mechanisms involving the reticular formation of all vertebrates are important for initiating the activation of behaviors (Pfaff et al., 2012). The hypothalamic cell cord along with a more caudal cell cord are reminiscent of such primitive structures. In the adult brain, structures along the cell cord serve synchronizing and patterning functions; $\mathrm{MCH}$ and the cognate hypocretin expressing neurons play roles in the sleep/wake cycle. MCH knockout mice have modified locomotor activities, and in humans, the absence of hypocretin in the dorsal hypothalamus is associated with narcolepsy (Peyron et al., 2000; Verret et al., 2003). This cell cord could also have important functions during all stages of brain development. Serotonergic neurons, which are among the very first generated cells in the hindbrain, act as pacemakers to synchronize the electrical activity of local motoneurons (Moruzzi et al., 2009). Later, the sot and tpoc are the precocious frames for the mfb. The early mfb contains dopamine and serotonin projections, and both neurotransmitters play key roles in the development of telencephalic structures. Dopamine modulates the cell cycle and proliferation in the ganglionic eminences and influences the maturation of the local circuitry (Diaz et al., 1997; Goffin et al., 2010). Serotonin has well recognized developmental effects. Alterations of early serotonergic or dopaminergic pathways lead to pathological conditions, such as autism or schizophrenia (Herlenius and Lagercrantz, 2001; Kinast et al., 2013). MCH is also suspected to have trophic actions (Cotta-Grand et al., 2009).

However, the second stage of forebrain development is more specifically associated with the differentiation of the hypothalamic medial regions and structures belonging to the 

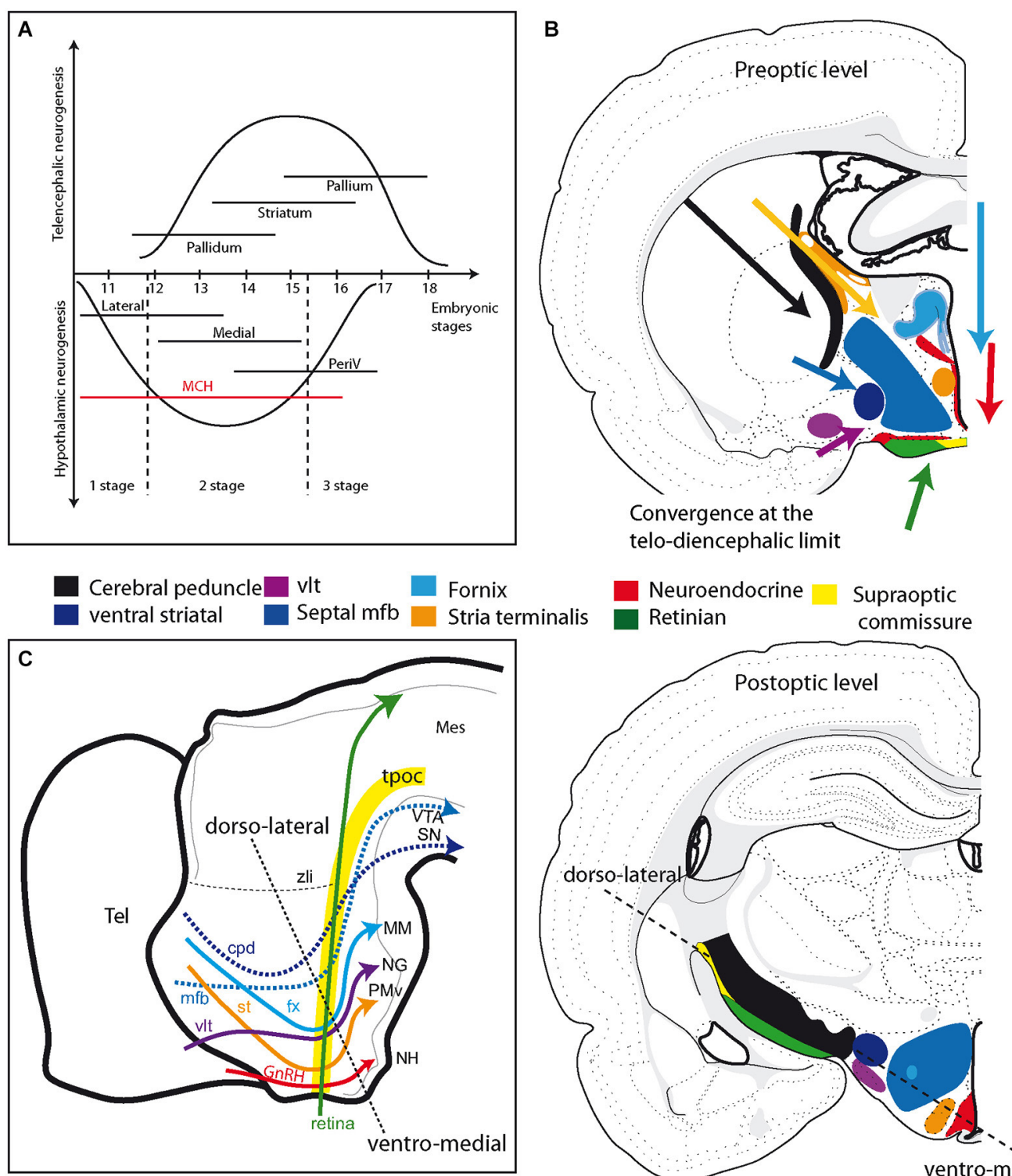

FIGURE 4 | (A) Schematic comparison of neurogenesis in the hypothalamus and telencephalon in the rat (see text for details). Neurogenesis in the hypothalamus is described as involving three stages: an early stage that produces only the lateral zone; a second that is concomitant to neurogenesis in the telencephalon and produces neurons in all hypothalamic longitudinal zones, but mostly the medial; a late third stage that concerns mainly periventricular zone neurons. Note that $\mathrm{MCH}$ neurons are produced during all three stages. (B) Illustration of the primary fiber tracts in the hypothalamus; these tracts originate in the telencephalon or retina and converge at the diencephalon-telencephalon limit at preoptic level (top drawing). In the postoptic hypothalamus (bottom drawing), these tracts are all aligned according to an axis determined by the dashed line. See text and supplementary information for details. (C) Schematic

classical striato-nigral pathways. These structures share genetic, topographic and chronotopic characteristics during development. This developmental stage coincides with the concept of a convergence in the anatomical organization of circuits involving these regions in the adult. It therefore becomes very attractive to describe the circuits connecting the pallium, striatum, pallidum and rostral brainstem as a series of parallel interacting loops (Figures 5B,C). Alexander described several putative circuits 



FIGURE 5 | (A) Schematic organization of olfactory and optic pathways in the hypothalamus compared to the cell cord. (B,C) Organization of the prosencephalic connectivity. The hypothalamus and ventral midbrain are engaged in circuit loops (blue in $\mathbf{B}$ ) that involve topographically organized descending inputs from the telencephalon and topographically organized outputs to the thalamus and tectum. These circuits play roles in behavioral expressions, voluntary motor responses and learning/memory. They are superimposed on a reticular core (including the VMPG), which is rostrally contiguous to the reticular brainstem, recalling the scaffold of the original cell cord, and are mostly involved with driving and patterning brain activities. See text for details. Abbreviations: Behav Cont Col: behavioral control column (medial zone nuclei of the hypothalamus); cer: cerebral; cpd: cerebral peduncle; ctx: cortex; fx: fornix; GnRH: gonadotropin-releasing hormone; LHA: lateral hypothalamic area; med: medial; mfb: medial forebrain bundle; $\mathrm{NH}$ : neurohypophysis; nuc: nucleus; olf: olfactory; SN: substantia nigra; st: stria terminalis; STN: subthalamic nucleus; TH: thalamus; VMPG: visceromotor pattern generator; VTA: ventral tegmental area; ZI: zona incerta. involving dorsal and ventral striatal components (Alexander et al., 1990). The Swanson group described several other circuits, involving medial and posterior striatal/pallidal structures and hypothalamic medial zone nuclei, suggesting the existence of a basic organizational plan (see Section Introduction; Swanson, 2003; Thompson and Swanson, 2010). We believe that we can now hypothesize that these sets of circuits are developmentally linked and can identify this as to be basic mammalian forebrain functional plan. Each of these circuits shows specific cytoarchitectonic characteristics and are either reticularly or nuclearly organized, likely under the control of the specific expression and localization of adherence molecules (CAM, cadherins) along the corresponding pathways (for example, for the nuclearly organized amygdala and medial hypothalamic nuclei connected through the stria terminalis). Some may even be characterized by the expression of specific transcription factors (again, as illustrated for the amygdala and hypothalamus, concerning reproductive and defensive pathways-Choi et al., 2005).

"Classic" authors have already suggested that well differentiated structures of highly organized brains must have emerged during evolution from primordial reticularly organized forms. Ramon-Moliner and Nauta used of the term "phylogenetic segregation" to characterize these evolutionary processes (RamónMoliner and Nauta, 1966). Pre- and postoptic hypothalamic structures have been observed in amphibians, as has a dopamine rich posterior tuberculum; however, laterally organized structures cannot be found in these species. Obviously, lateral and medial 
hypothalamic structures are phylogenetically recent but made of neurons that derivate from phylogenetically ancient populations (see in Croizier et al., 2013 for $\mathrm{MCH}$ and the dorsal hypothalamus), as are the substantia nigra/ventral tegmental area in the ventral midbrain. It is functionally relevant that these structures, including the mammillary nuclei (which contain head direction cells and are part of the Papez circuit), evolved in parallel. A larger behavioral repertory in mammals, especially related to reproductive and agonistic behaviors, is related to the differentiation of the hypothalamic medial and lateral zones, but is also likely associated with increased voluntary motor controls allowed by the extrapyramidal pathways.

To conclude, it has become obvious that the classical hypothalamus with its four regions does not constitute one single neurological entity, at least from the developmental point of view. The divergence in the origins of the collection of nuclei and areas that are usually gathered between its arbitrary borders can be traced to the earliest patterns of gene expression. However, here we contend that early neurogenesis gives rise to a first mantle layer, with longitudinal and transverse components (i.e., Y-shaped) that serves as a foundation for the formation of the whole forebrain connectivity, guiding most ascending and descending tracts appearing later. These observations demonstrate that the structures of the hypothalamic region are intimately implicated within complex networks along the extrapyramidal pathway and act together for the expression of behaviors. They also suggest that these circuits that involve the telencephalon and hypothalamus share a basic organizational plan.

\section{ACKNOWLEDGMENTS}

The authors are particularly grateful to Dr Gonzalo AlvarezBolado (Anatomisches Institut, Universität Heidelberg, Germany) and Philippe Ciofi (Institut Magendie, Bordeaux, France) for helpful discussions and careful reading of the manuscript.

\section{SUPPLEMENTARY MATERIAL}

The Supplementary Material for this article can be found online at: http://www.frontiersin.org/journal/10.3389/fnana. 2014.00161/abstract

\section{REFERENCES}

Aboitiz, F. (2011). Genetic and developmental homology in amniote brains. Toward conciliating radical views of brain evolution. Brain Res. Bull. 84, 125-136. doi: 10.1016/j.brainresbull.2010.12.003

Alexander, G. E., Crutcher, M. D., and Delong, M. R. (1990). Basal gangliathalamocortical circuits: parallel substrates for motor, oculomotor, "prefrontal" and "limbic" functions. Prog. Brain Res. 85, 119-146. doi: 10.1016/s00796123(08)62678-3

Altman, J., and Bayer, S. A. (1986). The development of the rat hypothalamus. Adv. Anat. Embryol. Cell Biol. 100, 1-178.

Alvarez-Bolado, G., Paul, F. A., and Blaess, S. (2012). Sonic hedgehog lineage in the mouse hypothalamus: from progenitor domains to hypothalamic regions. Neural Dev. 7:4. doi: 10.1186/1749-8104-7-4

Anderson, R. B., and Key, B. (1996). Expression of a novel N-CAM glycoform (NOC-1) on axon tracts in embryonic Xenopus brain. Dev. Dyn. 207, 263-269. doi: 10.1002/(sici)1097-0177(199611)207:3<263::aid-aja3>3.0. $\mathrm{co} ; 2-\mathrm{f}$
Bouret, S. G., Draper, S. J., and Simerly, R. B. (2004). Trophic action of leptin on hypothalamic neurons that regulate feeding. Science 304, 108-110. doi: 10. 1126/science.1095004

Brischoux, F., Cvetkovic, V., Griffond, B., Fellmann, D., and Risold, P. Y. (2002). Time of genesis determines projection and neurokinin-3 expression patterns of diencephalic neurons containing melanin-concentrating hormone. Eur. J. Neurosci. 16, 1672-1680. doi: 10.1046/j.1460-9568.2002. 02229.x

Brischoux, F., Fellmann, D., and Risold, P. Y. (2001). Ontogenetic development of the diencephalic $\mathrm{MCH}$ neurons: a hypothalamic ' $\mathrm{MCH}$ area' hypothesis. Eur. J. Neurosci. 13, 1733-1744. doi: 10.1046/j.0953-816x.2001. 01552.x

Brown, K. N., Chen, S., Han, Z., Lu, C. H., Tan, X., Zhang, X. J., et al. (2011). Clonal production and organization of inhibitory interneurons in the neocortex. Science 334, 480-486. doi: 10.1126/science.1208884

Canteras, N. S., Simerly, R. B., and Swanson, L. W. (1994). Organization of projections from the ventromedial nucleus of the hypothalamus: a Phaseolus vulgaris-leucoagglutinin study in the rat. J. Comp. Neurol. 348, 41-79. doi: 10. 1002/cne.903480103

Cheung, C. C., Kurrasch, D. M., Liang, J. K., and Ingraham, H. A. (2013). Genetic labeling of steroidogenic factor-1 (SF-1) neurons in mice reveals ventromedial nucleus of the hypothalamus (VMH) circuitry beginning at neurogenesis and development of a separate non-SF-1 neuronal cluster in the ventrolateral VMH. J. Comp. Neurol. 521, 1268-1288. doi: 10.1002/cne.23226

Choi, G. B., Dong, H. W., Murphy, A. J., Valenzuela, D. M., Yancopoulos, G. D., Swanson, L. W., et al. (2005). Lhx6 delineates a pathway mediating innate reproductive behaviors from the amygdala to the hypothalamus. Neuron 46, 647-660. doi: 10.1016/j.neuron.2005.04.011

Ciofi, P., Lapirot, O. C., and Tramu, G. (2007). An androgen-dependent sexual dimorphism visible at puberty in the rat hypothalamus. Neuroscience 146, 630642. doi: 10.1016/j.neuroscience.2007.02.028

Cotta-Grand, N., Rovère, C., Guyon, A., Cervantes, A., Brau, F., and Nahon, J. L. (2009). Melanin-concentrating hormone induces neurite outgrowth in human neuroblastoma SH-SY5Y cells through p53 and MAPKinase signaling pathways. Peptides 30, 2014-2024. doi: 10.1016/j.peptides.2009. 06.015

Croizier, S., Amiot, C., Chen, X., Presse, F., Nahon, J. L., Wu, J. Y., et al. (2011). Development of posterior hypothalamic neurons enlightens a switch in the prosencephalic basic plan. PLoS One 6:e28574. doi: 10.1371/journal.pone.00 28574

Croizier, S., Cardot, J., Brischoux, F., Fellmann, D., Griffond, B., and Risold, P. Y. (2013). The vertebrate diencephalic MCH system: a versatile neuronal population in an evolving brain. Front. Neuroendocrinol. 34, 65-87. doi: 10. 1016/j.yfrne.2012.10.001

Croizier, S., Franchi-Bernard, G., Colard, C., Poncet, F., La Roche, A., and Risold, P. Y. (2010). A comparative analysis shows morphofunctional differences between the rat and mouse melanin-concentrating hormone systems. PLoS One 5:e15471. doi: 10.1371/journal.pone.0015471

Cvetkovic, V., Brischoux, F., Griffond, B., Bernard, G., Jacquemard, C., Fellmann, D., et al. (2003). Evidence of melanin-concentrating hormonecontaining neurons supplying both cortical and neuroendocrine projections. Neuroscience 116, 31-35. doi: 10.1016/s0306-4522(02)00557-2

Diaz, J., Ridray, S., Mignon, V., Griffon, N., Schwartz, J. C., and Sokoloff, P. (1997). Selective expression of dopamine D3 receptor mRNA in proliferative zones during embryonic development of the rat brain. J. Neurosci. 17, 4282-4292.

Dominguez, L., Morona, R., González, A., and Moreno, N. (2013). Characterization of the hypothalamus of Xenopus laevis during development. I. The alar regions. J. Comp. Neurol. 521, 725-759. doi: 10.1002/cne.23222

Easter, S. S. Jr., Ross, L. S., and Frankfurter, A. (1993). Initial tract formation in the mouse brain. J. Neurosci. 13, 285-299.

Gelman, D., Griveau, A., Dehorter, N., Teissier, A., Varela, C., Pla, R., et al. (2011). A wide diversity of cortical GABAergic interneurons derives from the embryonic preoptic area. J. Neurosci. 31, 16570-16580. doi: 10.1523/jneurosci.4068-11. 2011

Goffin, D., Ali, A. B., Rampersaud, N., Harkavyi, A., Fuchs, C., Whitton, P. S., et al. (2010). Dopamine-dependent tuning of striatal inhibitory synaptogenesis. J. Neurosci. 30, 2935-2950. doi: 10.1523/jneurosci.4411-09. 2010 
Herlenius, E., and Lagercrantz, H. (2001). Neurotransmitters and neuromodulators during early human development. Early Hum. Dev. 65, 21-37. doi: 10. 1016/s0378-3782(01)00189-x

Herrick, C. J. (1910). The morphology of the forebrain in amphibian and reptilian. J. Comp. Neurol. 20, 413-547.

His, W. (1893). Vorschläge zur eintheilung des gehirns. Arch. Anat. Entwickel 3/4, $173-179$.

Keyser, A. (1972). The development of the diencephalon of the Chinese hamster. An investigation of the validity of the criteria of subdivision of the brain. Acta Anat. Suppl. (Basel) 59, 1-178.

Kinast, K., Peeters, D., Kolk, S. M., Schubert, D., and Homberg, J. R. (2013). Genetic and pharmacological manipulations of the serotonergic system in early life: neurodevelopmental underpinnings of autism-related behavior. Front. Cell. Neurosci. 7:72. doi: 10.3389/fncel.2013.00072

Kuhlenbeck, H. (1929). Die grundbestandteile des enddhirns im lichte der bauplanlehre. Anat. Anzig 67, 1-51.

Marín, O., Baker, J., Puelles, L., and Rubenstein, J. L. (2002). Patterning of the basal telencephalon and hypothalamus is essential for guidance of cortical projections. Development 129, 761-773.

Mastick, G. S., and Easter, S. S., Jr. (1996). Initial organization of neurons and tracts in the embryonic mouse fore- and midbrain. Dev. Biol. 173, 79-94. doi: 10. 1006/dbio.1996.0008

Mastick, G. S., Davis, N. M., Andrew, G. L., and Easter, S. S. Jr. (1997). Pax-6 functions in boundary formation and axon guidance in the embryonic mouse forebrain. Development 124, 1985-1997.

Moreno, N., and González, A. (2011). The non-evaginated secondary prosencephalon of vertebrates. Front. Neuroanat. 5:12. doi: 10.3389/fnana.2011. 00012

Moruzzi, A. M., Abedini, N. C., Hansen, M. A., Olson, J. E., and Bosma, M. M. (2009). Differential expression of membrane conductances underlies spontaneous event initiation by rostral midline neurons in the embryonic mouse hindbrain. J. Physiol. 587, 5081-5093. doi: 10.1113/jphysiol.2009. 180091

Nieuwenhuys, R., Geeraedts, L. M., and Veening, J. G. (1982). The medial forebrain bundle of the rat. I. General introduction. J. Comp. Neurol. 206, 49-81. doi: 10. 1002/cne.902060106

Nieuwenhuys, R., Ten Donkelaar, H. J., and Nicholson, C. (1998). The Central Nervous System of Vertebrates. Berlin: Springer-Verlag.

Nural, H. F., and Mastick, G. S. (2004). Pax6 guides a relay of pioneer longitudinal axons in the embryonic mouse forebrain. J. Comp. Neurol. 479, 399-409. doi: 10. 1002/cne.20317

Papez, J. W. (1995). A proposed mechanism of emotion. 1937. J. Neuropsychiatry Clin. Neurosci. 7, 103-112.

Perez-Balaguer, A., Puelles, E., Wurst, W., and Martinez, S. (2009). Shh dependent and independent maintenance of basal midbrain. Mech. Dev. 126, 301-313. doi: 10.1016/j.mod.2009.03.001

Peyron, C., Faraco, J., Rogers, W., Ripley, B., Overeem, S., Charnay, Y., et al. (2000). A mutation in a case of early onset narcolepsy and a generalized absence of hypocretin peptides in human narcoleptic brains. Nat. Med. 6, 991-997. doi: 10. $1038 / 79690$

Pfaff, D. W., Martin, E. M., and Faber, D. (2012). Origins of arousal: roles for medullary reticular neurons. Trends Neurosci. 35, 468-476. doi: 10.1016/j.tins. 2012.04.008

Pottin, K., Hinaux, H., and Rétaux, S. (2011). Restoring eye size in Astyanax mexicanus blind cavefish embryos through modulation of the Shh and Fgf8 forebrain organising centres. Development 138, 2467-2476. doi: 10.1242/dev. 054106

Puelles, L. (2001). Thoughts on the development, structure and evolution of the mammalian and avian telencephalic pallium. Philos. Trans. R. Soc. Lond. B Biol. Sci. 356, 1583-1598. doi: 10.1098/rstb.2001.0973

Puelles, L., Harrison, M., Paxinos, G., and Watson, C. (2013). A developmental ontology for the mammalian brain based on the prosomeric model. Trends Neurosci. 36, 570-578. doi: 10.1016/j.tins.2013.06.004

Puelles, L., Martinez-De-La-Torre, M., Bardet, S., and Rubenstein, J. L. R. (2012). "Hypothalamus," in The Mouse Nervous System, eds C. Watson, G. Paxinos and L. Puelles (London: Elsevier), 221-312.

Puelles, L., and Rubenstein, J. L. (2003). Forebrain gene expression domains and the evolving prosomeric model. Trends Neurosci. 26, 469-476. doi: 10.1016/s01662236(03)00234-0
Ramón-Moliner, E., and Nauta, W. J. (1966). The isodendritic core of the brain stem. J. Comp. Neurol. 126, 311-335. doi: 10.1002/cne.9012 60301

Rash, B. G., and Grove, E. A. (2011). Shh and Gli3 regulate formation of the telencephalic-diencephalic junction and suppress an isthmus-like signaling source in the forebrain. Dev. Biol. 359, 242-250. doi: 10.1016/j.ydbio.2011. 08.026

Ricaño-Cornejo, I., Altick, A. L., García-Peña, C. M., Nural, H. F., Echevarría, D., Miquelajáuregui, A., et al. (2011). Slit-Robo signals regulate pioneer axon pathfinding of the tract of the postoptic commissure in the mammalian forebrain. J. Neurosci. Res. 89, 1531-1541. doi: 10.1002/jnr. 22684

Riddle, R., and Pollock, J. D. (2003). Making connections: the development of mesencephalic dopaminergic neurons. Brain Res. Dev. Brain Res. 147, 3-21. doi: 10.1016/j.devbrainres.2003.09.010

Risold, P. Y. (2004). “The septal region,” in The Rat Nervous System, ed G. Paxinos (Amsterdam: Elsevier), 605-632.

Risold, P. Y., and Swanson, L. W. (1996). Structural evidence for functional domains in the rat hippocampus. Science 272, 1484-1486. doi: 10.1126/science.272.5267. 1484

Risold, P. Y., and Swanson, L. W. (1997). Connections of the rat lateral septal complex. Brain Res. Brain Res. Rev. 24, 115-195. doi: 10.1016/s0165-0173(97) 00009-X

Risold, P. Y., Thompson, R. H., and Swanson, L. W. (1997). The structural organization of connections between hypothalamus and cerebral cortex. Brain Res. Brain Res. Rev. 24, 197-254. doi: 10.1016/s0165-0173(97) 00007-6

Rubenstein, J. L., Shimamura, K., Martinez, S., and Puelles, L. (1998). Regionalization of the prosencephalic neural plate. Annu. Rev. Neurosci. 21, 445-477. doi: 10.1146/annurev.neuro.21.1.445

Shimamura, K., Hartigan, D. J., Martinez, S., Puelles, L., and Rubenstein, J. L. (1995). Longitudinal organization of the anterior neural plate and neural tube. Development 121, 3923-3933.

Shimogori, T., Lee, D. A., Miranda-Angulo, A., Yang, Y., Wang, H., Jiang, L., et al. (2010). A genomic atlas of mouse hypothalamic development. Nat. Neurosci. 13, 767-775. doi: 10.1038/nn.2545

Simerly, R. B. (2005). Wired on hormones: endocrine regulation of hypothalamic development. Curr. Opin. Neurobiol. 15, 81-85. doi: 10.1016/j.conb.2005. 01.013

Stoykova, A., Fritsch, R., Walther, C., and Gruss, P. (1996). Forebrain patterning defects in Small eye mutant mice. Development 122, 34533465 .

Suzuki, D. G., Murakami, Y., Escriva, H., and Wada, H. (2014). A comparative examination of neural circuit and brain patterning between the lamprey and amphioxus reveals the evolutionary origin of the vertebrate visual center. J. Comp. Neurol. 523, 251-261. doi: 10.1002/cne.23679

Swanson, L. W. (1987). "The hypothalamus," in Handbook of Chemical Neuroanatomy, Integrated Systems of the CNS, Part I, eds T. H. A. Björklund and L. W. Swanson (Amsterdam: Elsevier), 1-124.

Swanson, L. W. (2000). Cerebral hemisphere regulation of motivated behavior. Brain Res. 886, 113-164. doi: 10.1016/s0006-8993(00)02905-x

Swanson, L. W. (2003). Brain Architecture, Understanding The Basic Plan. New York: Oxford University Press.

Swanson, L. W. (2005). Anatomy of the soul as reflected in the cerebral hemispheres: neural circuits underlying voluntary control of basic motivated behaviors. J. Comp. Neurol. 493, 122-131. doi: 10.1002/cne.20733

Szabó, N. E., Zhao, T., Cankaya, M., Theil, T., Zhou, X., and AlvarezBolado, G. (2009). Role of neuroepithelial Sonic hedgehog in hypothalamic patterning. J. Neurosci. 29, 6989-7002. doi: 10.1523/jneurosci.1089-09. 2009

Thompson, R. H., and Swanson, L. W. (2003). Structural characterization of a hypothalamic visceromotor pattern generator network. Brain Res. Brain Res. Rev. 41, 153-202. doi: 10.1016/s0165-0173(02)00232-1

Thompson, R. H., and Swanson, L. W. (2010). Hypothesis-driven structural connectivity analysis supports network over hierarchical model of brain architecture. Proc. Natl. Acad. Sci. U S A 107, 15235-15239. doi: 10.1073/pnas. 1009112107

Verret, L., Goutagny, R., Fort, P., Cagnon, L., Salvert, D., Léger, L., et al. (2003). A role of melanin-concentrating hormone producing neurons in the central 
regulation of paradoxical sleep. BMC Neurosci. 4:19. doi: 10.1186/1471-22024-19

Vitalis, T., and Rossier, J. (2011). New insights into cortical interneurons development and classification: contribution of developmental studies. Dev. Neurobiol. 71, 34-44. doi: 10.1002/dneu.20810

Ye, W., Shimamura, K., Rubenstein, J. L., Hynes, M. A., and Rosenthal, A. (1998). FGF and Shh signals control dopaminergic and serotonergic cell fate in the anterior neural plate. Cell 93, 755-766. doi: 10.1016/s0092-8674(00)81437-3

Conflict of Interest Statement: The authors declare that the research was conducted in the absence of any commercial or financial relationships that could be construed as a potential conflict of interest.
Received: 30 September 2014; accepted: 09 December 2014; published online: 06 January 2015.

Citation: Croizier S, Chometton S, Fellmann D and Risold P-Y (2015) Characterization of a mammalian prosencephalic functional plan. Front. Neuroanat. 8:161. doi: 10.3389/fnana.2014.00161

This article was submitted to the journal Frontiers in Neuroanatomy.

Copyright $\odot 2015$ Croizier, Chometton, Fellmann and Risold. This is an open-access article distributed under the terms of the Creative Commons Attribution License (CC $B Y)$. The use, distribution and reproduction in other forums is permitted, provided the original author(s) or licensor are credited and that the original publication in this journal is cited, in accordance with accepted academic practice. No use, distribution or reproduction is permitted which does not comply with these terms. 